\title{
ANALYSIS OF THE WEAR PROCESS OF THE ELEMENTS IN THE BRAKING SYSTEM OF HEAVY TRUCKS
}

\author{
Mădălin-Florin POPA, Bogdan Manolin JURCHIȘ \\ Technical University of Cluj-Napoca, Romania \\ e-mail: madalin.popa@live.com
}

\begin{abstract}
Today's commercial vehicles work in a very competitive environment, in an extremely active economic market and the requirements for the delivered performance make it imperative to study in detail each component element. In practice, all these aspects are found in the process of wearing the elements of different systems. Moreover, major changes are expected for the near future of motor vehicles in order to improve the ecological balance both by reducing the pollutant emissions of the propellants and by improving the percentage of recyclability and reduction of waste from maintenance (liquids and solutions such as oils, antifreeze or solutions with different detergents). In order to identify solutions to improve the mentioned problems, the current study analyses the types of wear identified in the elements of the braking system of commercial vehicles and their main factors of influence. The study is based on data obtained during analysis during operation for a number of trucks with different configurations.
\end{abstract}

KEYWORDS: brake, wear, brake pads wear, brake disk wear

\section{Introduction}

In order to be competitive, commercial vehicles must be able to carry heavy loads in the shortest possible time and very importantly, with minimal costs. The current braking systems that equip this type of vehicle are widely used and offer extremely good performance and low costs compared to previous generations. Until they are replaced with new variants of braking systems that allow in addition to high braking performance and total or partial energy recovery, improving performance and reducing maintenance costs remain two topics of major importance.

The identification of the impact that a number of external and operating factors have on the braking system as well as the analysis of the wear of the elements in the friction torque are also study elements that outline the main purpose of this study. It should be noted that this study provides an analysis based on the direct study of trucks operating in real working conditions. Moreover, extreme wear and tear situations resulting from over-stresses were also analysed.

Identification and analysis of problems during operation, because they are the starting point of experimental research. Occasionally, the problems of each system must be analysed and we can mention here systems such as: electrical, mechanical, hydrodynamic, main and auxiliary systems, etc. The analysis of each system with its particularities requires a large volume of data to be analysed, but based on them, conclusive and efficient solutions can be provided for the current major problems of motor vehicles. It should be noted that the paper seeks to address the analysis of situations encountered in operation from several perspectives. The proposal of solutions for the identified problems also touches on topics related to: materials used in the friction torque, constructive variants for the braking systems, the mode of operation and even aspects related to the problem of obsolete parts.

The analysis of the interventions in the service of heavy trucks led to the conclusion that they generate a large volume of used parts, which do not find their place in a well-developed recycling system. Another issue that needs to be clarified and requires increased attention is the decisive factor that determines the need to replace a part. In the absence of advanced systems for accurately measuring wear, many parts are replaced prematurely and others are replaced too late, leading to damage to other parts of the system, thus entering a vicious circle. 


\section{Elements of analysis}

One of the essential conditions for a braking system to deliver the specified performance is that its elements meet certain technical conditions. In addition to the assemblies, an essential factor is the quality of the parts. A good quality part will satisfy a range of use as accurately as possible, without causing unwanted wear to other parts. Wear elements are the brake pads, which are most often replaced due to wear and tear after friction with the brake disc. The latter is a more expensive part and more difficult to access, so it will have to withstand a longer period of operation. In addition to wear on discs and pads, defects are very common in the sealing gaskets either in the pistons in the brake calliper or in the sliding bolts in the brake callipers. Frequent damage caused by corrosion has also been found in vehicles older than 10 years.

All these defects must be prevented in such a way that it is not possible to carry out the braking process while driving, so that there must be regular intervals of preventive inspection. More recently, electronic assistance systems have been used to monitor system parts. However, at present, the only maintenance system for the brake system is based on the information transmitted to the brake pad wear sensor display (Figure 1). Based on this, service interventions will be required to replace the brake pads when the electronic system alerts the driver.

The wear and tear of discs and pads will be assessed by authorized workshop personnel on the basis of standards. However, this process can be subjective and inefficient and it would be preferable to have an automatic wear monitoring system or to identify new solutions, which would allow greater control over the wear phenomena in the friction torque.

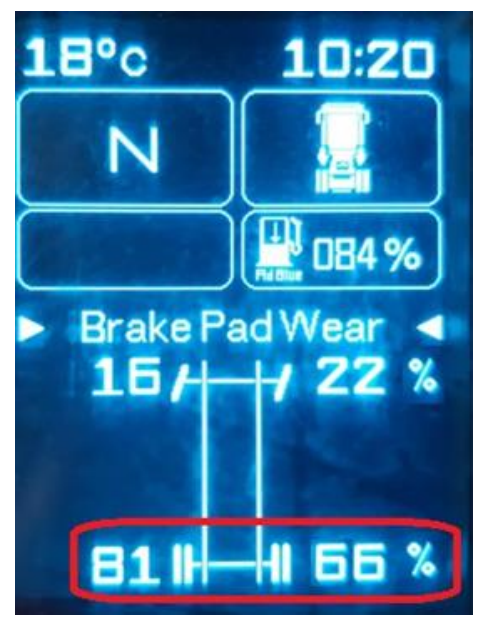

Fig. 1. Information provided to the user regarding the stage of wear of the brake pads

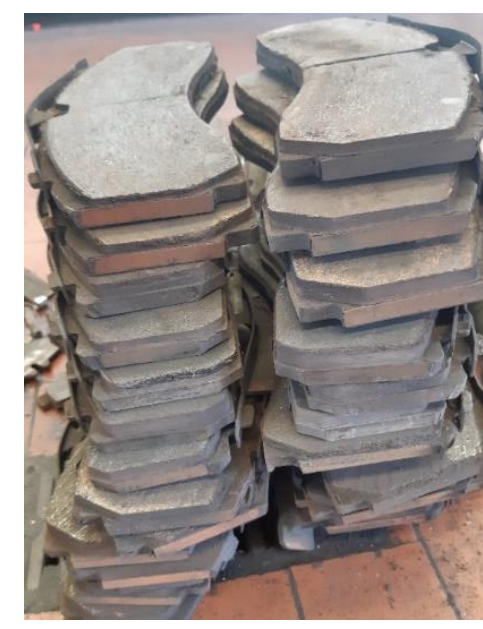

Fig. 2. Large volume of obsolete parts that do not have a recycling plan

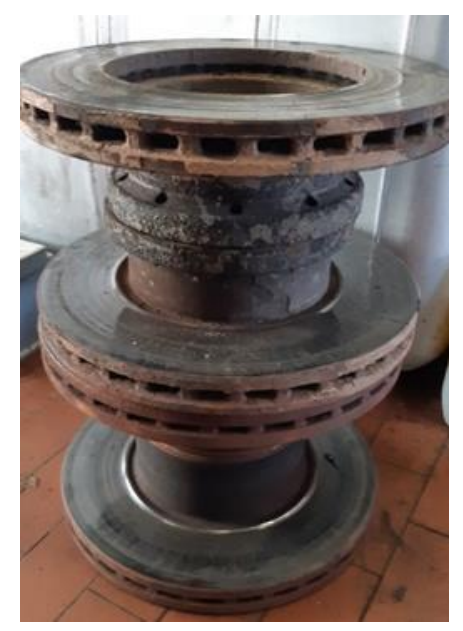

Fig. 3. Worn brake discs for which there is no recycling plan

\section{Analysis of identified types of wear}

\subsection{Normal wear of the elements of the friction torque of the main braking system}

All interventions for replacements to the braking system, apart from the maintenance program initially established, represent abnormal wear. These have different causes and, in the literature, there are a number of papers that address these issues [1-5]. Friction between the plate and the disc will result in a change in the size of the two parts. Normally, the wear elements are the friction linings of the brake pads. The reduction of their dimensions is done up to a certain maximum accepted value. Slowing down the wear process and identifying solutions by using new materials are elements frequently analysed in the literature [6-8]. 
Figures 2 and 3 show parts replaced during a calendar month in the repair shop where the analysis was performed. There is a very large volume of replaced brake pads and a few worn brake discs. Among the parts in both figures can be seen some that have not yet reached the degree of wear but are still replaced. These issues related to the premature replacement of some elements require a careful analysis both for the efficiency of the economic balance and for the identification of solutions for electronic monitoring and evaluation of wear elements in order to determine the exact degree of wear and use of resources as efficiently as possible.

\subsection{Excessive wear of the elements in the braking system}

Braking efficiency is greatly influenced by the condition of the final brake effectors (brake calliper, brake pads, discs or brake shoes). Continuous monitoring of the condition of these elements increases traffic safety by continuously knowing the degree of wear of the parts in the system. However, the study [9] identifies and analyses a number of external factors, encountered in operation, which cause serious damage to the braking systems by the effect on the parts intended for maintenance control or by the direct damage of the brake pads or discs under conditions. extreme. In practice, the main problems that lead to excessive wear are caused by [9]: blockage of the brake callipers due to dust and mud on the road; suppression of the movement of the brake pads; non-compliant parts; overloads; uneven and unforeseen wear and tear; aggressive driving style.

Aspects related to the wear profile of certain sets of brake pads should also be considered. This can lead to the identification of areas of wear, on the basis of which an overview of wear can be outlined. These studies, unlike those performed on brake pads, reveal unforeseen issues encountered only in real operating conditions and the results can bring significant improvements, both in terms of performance and system reliability.

After analysing the wear intervals (by reading the values from the brake pad wear sensors), from the total number of trucks analysed, at $8.24 \%$ of them, at least a wear value greater than $99 \%$ was identified for one of the brake pads. The distribution of these cases (Figure 4) was analysed as in the other cases based on the current maintenance interval. As already mentioned, in the interval 4 the data cannot be completely analysed due to the large number of kilometres required to be covered until the beginning of the interval and then for the passage of the interval until the total wear. The "interval" represented the distance travelled between two brake pad replacements.

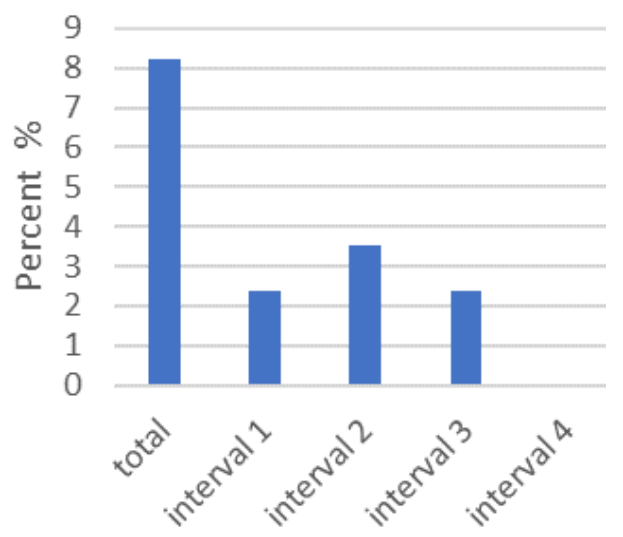

Fig. 4. Cases with wear greater than $99 \%$

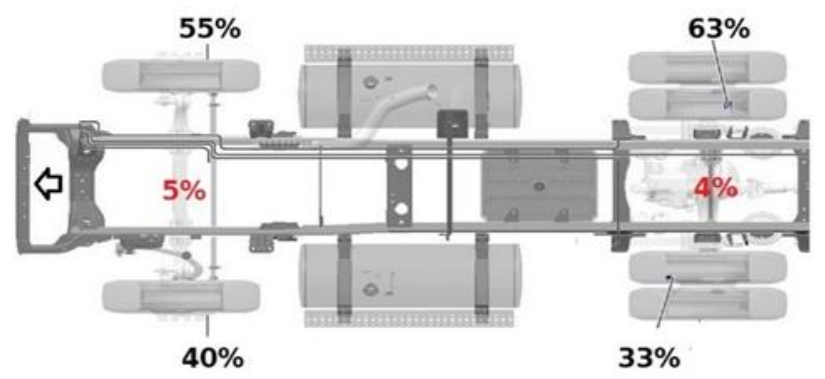

Fig. 5. Cases in which there is a greater wear of the brake elements on the right wheel

\subsection{Different wear of the brake pads of the same axle}

The study also carefully analysed the frequency of cases in which differentiated wear of the elements in the friction torque occurs. In general, there have been significantly more cases with more wear on the right-hand brake elements. Figure 5 shows the percentages in which the wear was higher on the respective wheel and in the middle are the percentages in which the wear was equal to the wheels of the same axle (with an error of $1 \%$ ). In $55 \%$ 
of cases on the front axle and in $63 \%$ of cases on the rear axle there was more wear on the right side, while only in $5 \%$ of cases on the front axle and $4 \%$ of cases on the rear axle respectively, no there were differences in wear.

\subsection{Different wear of the brake pad on the inside than on the outside}

Analysing the shape of the wear, it was found that a determining factor for the premature replacement of the brake pads or discs is due to the uneven wear of the elements in the system. This can be either a different wear of the brake pad from the inside than the outside or another case can be due to the different wear of the pads on the wheels of the same axle. Figure 6 shows an example of different wear of the outer pad from the inside and Figure 7 shows the effect of different wear on the brake pads.

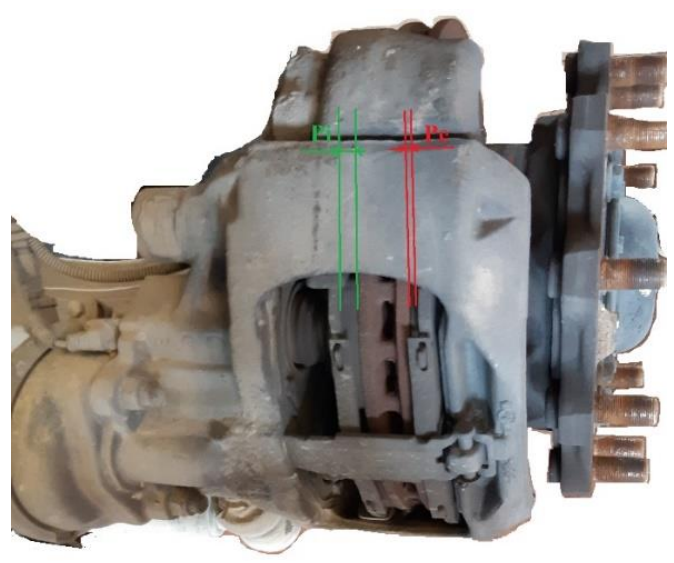

Fig. 6. Wear difference on the inner brake pad (Pi) compared to the outer brake pad (Pe)

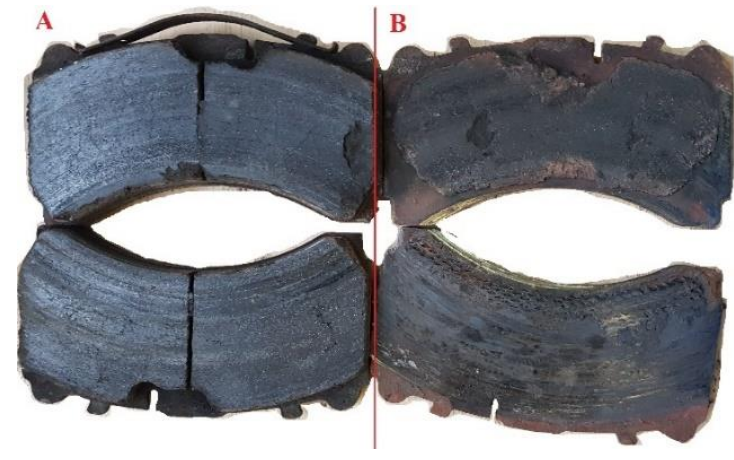

Fig. 7. Brake pads of the same axle, worn differently (A-left; B-right)

In both cases of wear and tear, the cause of the wear and tear was the suppression of the calliper or the plate. Specifically, Figure 6 shows the locking movement of the brake pad from the outside relative to its support, and Figure 7 shows the difference in wear between the left and right is caused by the locking movement of the left calliper on the bolts its. Without the calliper sliding, the braking on the axle was performed differently, which only led to the wear of the elements in the functional brake coupling. The operation of the calliper sliding system was discussed in the first part of the thesis. The translational movement of the calliper is absolutely necessary so that the pressing force of the pistons is applied uniformly on the surface of both brake pads.

\subsection{The influence of external factors on brake wear}

Suppression of the friction torque elements is a common cause of premature replacement of parts in the system. During the study it was found that although there is a complex maintenance system, this type of defect is not monitored electronically. Such a brake calliper position and movement monitoring system would be useful in this regard but would bring additional costs to the system. In this case, the question is whether these additional costs are lower than the damage caused by defects and whether a cheap system could be implemented to streamline the process.

The analysis of the factors that favour these defects is an essential element for the improvement of the performances, at the same time as the reduction of the maintenance costs. Figure 8 shows one of the external factors that most frequently contributes to blocking the translational motion, namely deposits of dust, mud and other materials from the outside environment.

During the analysis, a series of defects were identified caused by impurities from the external environment, the presence of which favors abrasion wear. In addition to the influence of abrasive materials from the outside environment, corrosion is also a major problem because there are a number of common factors that influence wear and tear and significantly reduce the life of the elements in the braking system. The combination of several types of wear based on common influencing factors generates defects such as those in Figure 9.

The main effects of the situations shown in Figure 9 lead to increased degradation and premature decommissioning of the pads and (in most cases) brake discs, leading to a significant increase in maintenance costs. As a side effect of these types of wear, there are a number of specific noises (rumble or sharp noises). The intensity and type of these noises significantly affect the quality of life in areas close to roads. At the same time, these noises are the main warning about the occurrence or existence of a fault. 
A

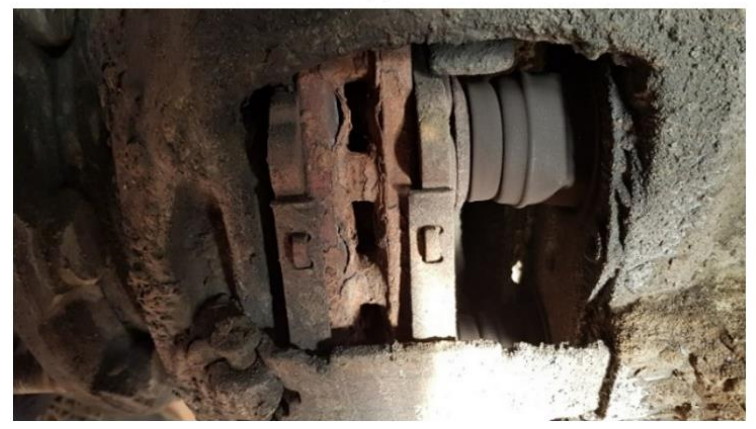

B

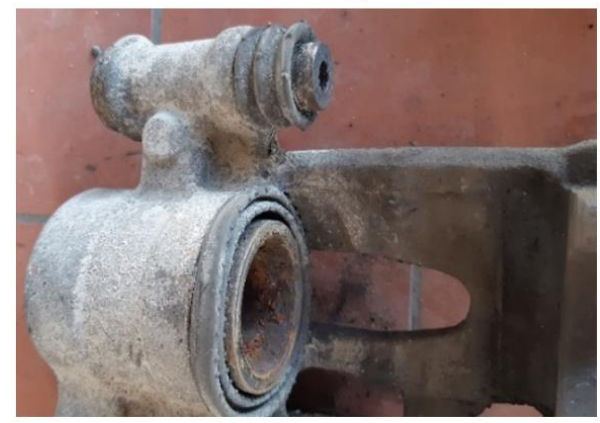

Fig. 8. Deposits and corrosion under normal working conditions A-deposits on the stirrup; Bdeposits on the guide bolts of the brake calliper

A.

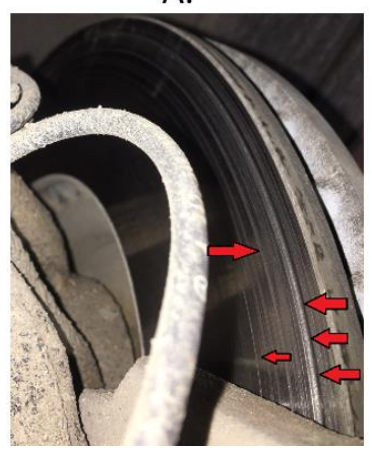

B.

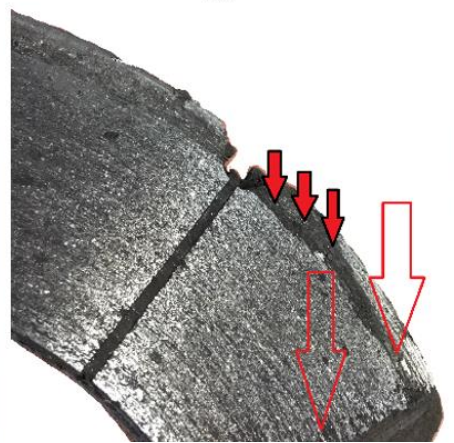

C.

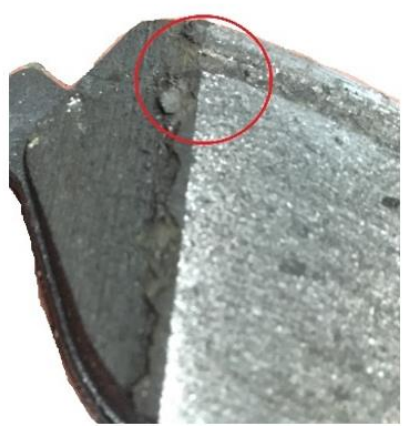

D.

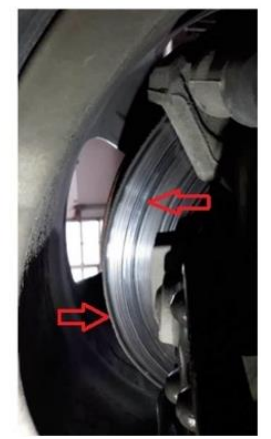

Fig. 9. Combined effects of the influence of impurities and particles from the external environment on the wear of the brake discs and pads: A, D- the effects of abrasive wear on the brake disc; $B, C$ degradation of the brake pads under the action of external factors and corrosion

\section{Analysis of distances travelled until the replacement of the brake pads}

Figure 10 shows the situation of another truck without an auxiliary braking system, which is running in internal traffic but which already has up to 477000 $\mathrm{km}$ and has 4 brake pad replacements. This is a significant justification for the need to use auxiliary braking systems. During the work, several cases of accentuated wear of the elements in the braking system were found and frequent thermal overloads of the brakes were frequently identified with serious effects on the braking performance. It has also been found that when heavy wear occurs or in cases of thermal overload, repair costs increase significantly and, in most cases, it becomes necessary to replace the brake discs. The current study also contains an economic analysis of these maintenance costs for certain cases but also a part of the analysis of the effects of thermal overloads on the elements of the braking system.

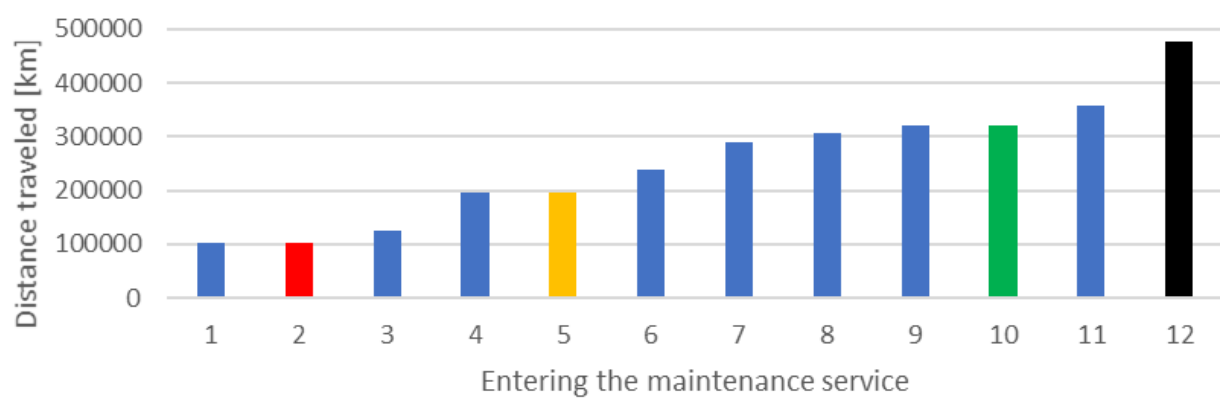

Fig. 10. Distribution of service entries and replacement of brake pads on the front axle to a truck without an auxiliary braking system 
In the case of extreme wear and tear, it has been found that exceeding the maintenance intervals or exceeding the maximum wear limits comes with additional costs caused in many cases and the need to replace the brake discs. In order to avoid these situations, the evolution of wear and tear and the distribution of cases in which wear is close to the maximum values were studied.

The graphs in Figures 11 and 12 show the distribution of cases with wear greater than $95 \%$. The degree of wear is read from the data taken from the brake pad wear sensors.
It is observed that both the front and the rear axle have a high weight in cases where the wear is higher than 95\%. The technical documentation provided by the manufacturer recommends replacement from $90 \%$ (even $85 \%$ in some cases) so further use from values above $95 \%$ is not recommended. Above these values, the rate of serious defects increases exponentially, so the continued use is not only no longer justified but also with much higher risks.

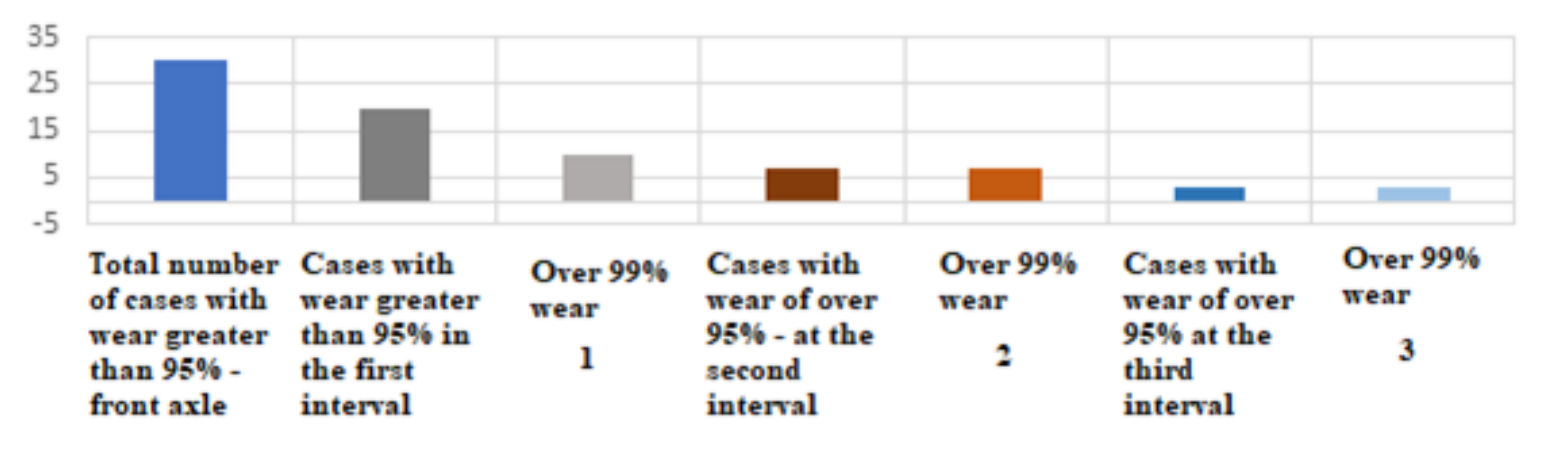

Fig. 11. Distribution of wear cases close to the maximum limit for the front axle

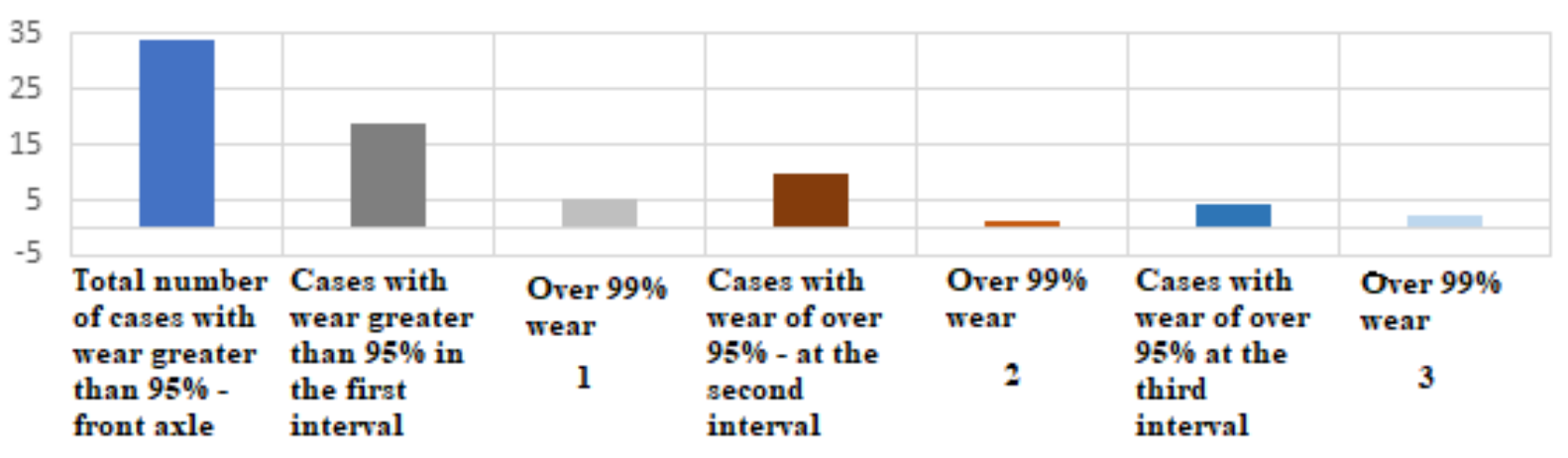

Fig. 12. Distribution of wear cases close to the maximum limit for the rear axle

\section{Aspects and problems related to waste management resulting from the wear of the elements during braking}

Figure 13 shows the parts replaced during a calendar month in the repair shop where the analysis was performed. There is a very large volume of replaced brake pads and a few worn brake discs. Among the parts, you can see some that have not yet reached the degree of wear but are still replaced. These issues related to the premature replacement of some elements require a careful analysis both for the efficiency of the economic balance and for the identification of solutions for electronic monitoring and evaluation of the elements of wear in order to determine the exact degree of wear and the most efficient use of resources.

Following the analysis of the problems encountered in operation on commercial vehicles, it was found that in addition to the problems of wear and defects, these vehicles also generate a large volume of waste resulting from the parts used. This aspect needs special attention, especially in the ecological context of environmental protection. In this sense, a major regulation of these situations would be useful, especially since these used parts are generated, as it was found, in quite large quantities (Fig. 13).

The production of a small volume of waste can be controlled from the period of use, when the main causes that cause premature or accentuated defects can be established and solutions can be identified to 


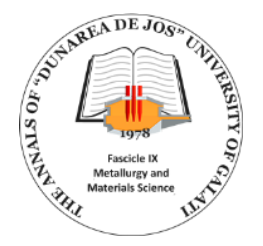

\section{THE ANNALS OF “DUNAREA DE JOS” UNIVERSITY OF GALATI \\ FASCICLE IX. METALLURGY AND MATERIALS SCIENCE \\ $\mathrm{N}^{\circ} .4$ - 2021, ISSN 2668-4748; e-ISSN 2668-4756 \\ Article DOI: https://doi.org/10.35219/mms.2021.4.13}

improve the processes. Thus, the analysis of braking systems becomes a component part of the environmental and quality of life problems, not only

by the volume of waste generated but also by the direct effects of the noise produced by braking on people in the immediate vicinity of the roads.
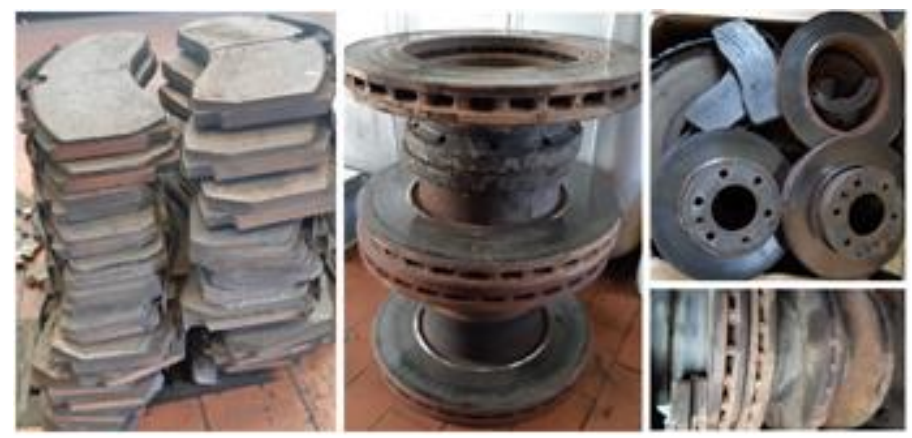

Fig. 13. The problem of used parts that generate a large volume of waste and for which there is no recycling plan

\section{Conclusions}

Starting from the analysis of operational defects, it can be concluded that, in addition to the normal wear problems, there are a number of different wear situations, the effects of which can cause a drastic decrease in traffic safety and additional operating costs. In this context, the analysis of factors favouring wear and tear is a key element in increasing traffic safety and reducing additional operating costs.

The main problems identified in the operation refer to: the generation of a large volume of residual parts; premature replacement of parts; subjectivity to the decision to replace parts, without being monitored by a precise maintenance system; uneven wear of brake discs (shoulder wear); uneven wear of brake pads; deposits that suppress the freedom of movement of some elements of the friction torque; advanced corrosion in different areas; parts failures and serious defects identified as a result of overloading in heavy site conditions.

\section{References}

[1]. Vdovin A., et al., A coupled approach for vehicle brake cooling performance simulations, International Journal of Thermal Sciences, 132, p. 257-266, 2018.

[2]. Hatam A., Khalkhali A., Simulation and sensitivity analysis of wear on the automotive brake pad, Simulation Modelling Practice and Theory, 84, p. 106-123, 2018.

[3]. Elgharbawy M., et al., Adaptive functional testing for autonomous trucks, International Journal of Transportation Science and Technology, December 2018.

[4]. Blau P. J., Jolly B. C., Wear of truck brake lining materials using three different test methods, Wear, 259, p. 1022-1030, 2005.

[5]. Adamowicz A., Grzes P., Influence of convective cooling on a disc brake temperature distribution during repetitive braking, Therm. Eng., 31, p. 2177-2185, 2011.

[6]. Wahlström J., Olander A. S. L., Jansson A., Olofsson U., A pin-on-disc simulation of airborne wear particles from disc brakes, Wear, 268, p. 763-769, 2010.

[7]. Shiva Shanker P., A review on properties of conventional and metal matrix composite materials in manufacturing of disc brake, Mater. Today Proc., 5, p. 5864-5869, 2018.

[8]. Poletto J. C., et al., Analysis of the error in the estimation of the morphology of contact plateaus existing on the surface of brake pads, Tribology International, 126, p. 297-306, 2018.

[9]. Popa M. F., Capătă D. M. S., Burnete N., Aspects of wear braking process on brake discs and brake pads in the construction of commercial vehicles, Fascicle of Management and Technological Engineering, May 2019. 